\title{
Modeling the Effect of Decreasing Dissolved Oxygen on Fish Population Survival in Aquatic Body in the Presence of Nutrients
}

\author{
Shikha Chaturvedi ${ }^{1}$, , Prabha S. Rastogi ${ }^{2}$ \\ ${ }^{1}$ Fr. C. Rodrigues Institute of Technology, Vashi, Maharashtra, India. \\ 2 Shri J.J.T. University, Jhunjhunu, Rajasthan, India. \\ * Corresponding author. Tel.: +91-02241611000; email: cshikha11@gmail.com \\ Manuscript submitted October 12, 2015; accepted January 4, 2016. \\ doi: 10.17706/ijapm.2016.6.1.9-16
}

\begin{abstract}
In this paper a nonlinear mathematical model is proposed to analyze the effect of decreasing level of dissolved oxygen on the survival of aquatic species in the water body in the presence of nutrients. This model has four nonlinear differential equations consisting of four state variables accounting for concentration of nutrients, density of algae, density of fish population, and concentration of dissolved oxygen. The three equilibrium points have been obtained to understand the dynamic behavior of the system. The conditions for existence of these equilibrium points are obtained. The stability analysis is conducted on each equilibrium point by considering variational matrix and using Routh-Hurwitz criteria. The nonlinear stability behavior of an equilibrium point considered to be critical is analyzed by applying Liapunov's direct method.
\end{abstract}

Key words: Algae, dissolved oxygen, Liapunov's function, mathematical model, nutrients.

\section{Introduction}

The water runoff from the farmlands and factories contains pollutants in the form of fertilizers and organic matters. These pollutants have nutrients such as nitrogen, phosphorus, carbon etc. The nutrient rich chemicals help the aquatic plants to grow better as they do for agricultural plants. The excessive amount of these nutrients causes excessive growth of algae resulting in the depletion of dissolved oxygen (DO) which in turn causes threat to the survival of aquatic species. The research work in this area started in the year 1925. Streeter and Phelps [1] in 1925 proposed the first dynamical model on depleting DO due to discharge of organic pollutants. Further Dobbins [2] and O' Corner [3] generalized this model. Many researchers [4]-[8] studied depletion of DO by various sources and the survival of aquatic species. Arnold and Voss [9] studied numerical behavior of eutrophied lakes. Marler and Westfield [10] discussed a mathematical model based on ecological problem in which depletion of DO occurs due to growth of bacteria and protozoa. Shukla et al. [11], [12] studied the existence and survival of two competing species in a polluted water body and effect of changing habitat on survival of aquatic species.

Phytoplankton also plays an important role in the dynamics of aquatic body. Khare et al. [13], [14] discussed the effect of the depleting DO on the survival of interacting planktonic population. However they didnot consider the crowding effect factor in their analysis. Many investigators [15] carried out modeling studies involving zooplankton, phytoplankton and nutrients. However they did not consider the 
concentration of DO in the model. Keeping in view these aspects, in the present study a mathematical model is proposed to estimate the effect of decreasing DO on fish population survival in aquatic body in the presence of nutrients. It accounts for concentration of nutrients, density of algae, density of fish population, and concentration of DO. Analysis of each equilibrium point is carried out in which the existence criterion of each point is shown. Further, the stability behavior of these points is discussed by using Routh-Hurwitz criteria. Then the nonlinear stability behavior of the critical point is studied by using Liapunov's direct method. To substantiate the feasibility of the model, numerical simulation is carried out.

\section{Mathematical Model}

Let $N$ be the cumulative concentration of nutrients, $A$ be the density of algae, $C$ be the concentration of DO, and $F$ be the density of fish populations. These are the four state variables. Further let us consider $Q$ be the cumulative rate of discharge of nutrients, $q$ be the rate of growth of DO by various sources. It is further assumed that the growth rate of algae is proportional to the term $\left[N A / \alpha_{1}+C_{0}-C\right]$. Let $v_{2}, v_{3}$, and $v_{4}$ are the natural depletion rate of algae, concentration of DO and fish population respectively. Predation rate of algae by fish is $\alpha_{2}$. It is assumed that the growth rate of fish population is proportional to the term $\left[A F / \alpha_{4}+C_{0}-C\right]$. Here $\alpha_{1}$ and $\alpha_{4}$ are assumed to be half saturation constants, $C_{0}$ is the saturation value of DO and $C_{0}-C$ is oxygen deficit.

Considering the above facts, the system is governed by following nonlinear ordinary differential equations.

$$
\begin{gathered}
\frac{d N}{d t}=Q-v_{1} N-\beta_{1} N A, \\
\frac{d A}{d t}=\frac{\beta_{2} N A}{\alpha_{1}+C_{0}-C}-v_{2} A-\alpha_{2} A F, \\
\frac{d C}{d t}=q-v_{3} C, \text { and } \\
\frac{d F}{d t}=\frac{\alpha_{3} A F}{\alpha_{4}+C_{0}-C}-v_{4} F .
\end{gathered}
$$

Here $v_{1}, v_{2}, v_{3}$, and $v_{4}$ are depletion rate coefficients and $\beta_{1}, \beta_{2}, \alpha_{2}$, and $\alpha_{3}$ are positive proportionality constants. It is important to note here that the initial value of each parameter is positive i.e. $N(0)=N_{10}>0$, $A(0)=A_{10}>0, C(0)=C_{10}>0$, and $F(0)=F_{10}>0$.

\section{Analysis of equilibrium points of the system}

The model discussed in the above section has following three nonnegative equilibrium points.

1) Initially, it is considered that there is no change in the rate of flow of nutrients and concentration of DO, thus $d N / d t=0$ and $d C / d t=0$ which give the terms $Q / v_{1}$ and $q / v_{3}$. The $1^{\text {st }}$ equilibrium point $E_{1}$ is obtained which always exists and given by $E_{1}\left(Q / v_{1}, 0, q / \nu_{3}, 0\right)$.

2) The $2^{\text {nd }}$ equilibrium point is given by $E_{2}\left(N^{\prime}, A^{\prime}, C^{\prime}, 0\right)$.

where $N^{\prime}=\frac{v_{2}}{\beta_{2} v_{3}}\left(\alpha_{1} v_{3}+C_{0} v_{3}-q\right), C^{\prime}=\frac{q}{v_{3}}$, and $A^{\prime}=\frac{Q \beta_{2} v_{3}-v_{1} v_{2}\left(v_{1} v_{3}+C_{0} v_{3}-q\right)}{\beta_{1} v_{2}\left(\alpha_{1} v_{3}+C_{0} v_{3}-q\right)}$. 
Thus $E_{2}$ exists, provided the following conditions are satisfied:

$$
\begin{gathered}
\alpha_{1} v_{3}+C_{0} v_{3}-q>0, Q \beta_{2} v_{3}-v_{1} v_{2}\left(\alpha_{1} v_{3}+C_{0} v_{3}-q\right)>0, v_{4}\left(\alpha_{4}+C_{0}-C^{\prime}\right)-\alpha_{3} A^{\prime}>0, \\
\alpha_{1}+C_{0}-C^{\prime}>0, \text { and } Q-v_{1} N^{\prime}>0 .
\end{gathered}
$$

3) The $3^{\text {rd }}$ equilibrium point is given by $E_{3}\left(N^{*}, A^{*}, C^{*}, 0\right)$, where

$$
\begin{gathered}
C^{*}=\frac{q}{v_{3}}, A^{*}=\frac{v_{4}\left(\alpha_{4} v_{3}+C_{0} v_{3}-q\right)}{\alpha_{3} v_{3}}, \\
N^{*}=\frac{Q \alpha_{3} v_{3}}{\left[v_{1} v_{3} \alpha_{3}+\beta_{1} v_{4}\left(\alpha_{4} v_{3}+C_{0} v_{3}-q\right)\right]},
\end{gathered}
$$

and

$$
F^{*}=\frac{Q \alpha_{3} v_{3}^{2} \beta_{2}}{\alpha_{2}\left[\alpha_{1} v_{3}+C_{0} v_{3}-q\right]\left[v_{1} v_{3} \alpha_{3}+\beta_{1} v_{4}\left(\alpha_{4} v_{3}+C_{0} v_{3}-q\right)\right]}-\frac{v_{2}}{\alpha_{2}} .
$$

Thus $E_{3}$ exists if these conditions are satisfied:

$$
\alpha_{4} v_{3}+C_{0} v_{3}-q>0, \beta_{2} N^{*}-v_{2}\left(\alpha_{1}+C_{0}-C^{*}\right)>0,\left(\alpha_{1}+C_{0}-C^{*}\right)>0, \text { and }\left(\alpha_{4}+C_{0}-C^{*}\right)>0
$$

\section{Stability Analysis}

In this section the stability behavior of each equilibrium point is discussed. The variational matrix $J_{\mathrm{i}}$ of The system"(1)-(4)" is given as follows :

$$
J_{\mathrm{i}}=\left[\begin{array}{cccc}
-v_{1}-\beta_{1} A & -\beta_{1} N & 0 & 0 \\
\frac{\beta_{2} A}{\alpha_{1}+C_{0}-C} & \frac{\beta_{2}{ }^{N}}{\alpha_{1}+C_{0}-C}-v_{2}-\alpha_{2} F & \frac{\beta_{2} N A}{\left(\alpha_{1}+C_{0}-C\right)^{2}} & -\alpha_{2} A \\
0 & 0 & -v_{3} & 0 \\
0 & \frac{\alpha_{3} F}{\alpha_{4}+C_{0}-C} & \frac{\alpha_{3} A F}{\left(\alpha_{4}+C_{0}-C\right)^{2}} & \frac{\alpha_{3} A}{\alpha_{4}+C_{0}-C}-v_{4}
\end{array}\right]
$$

\subsection{Case 1: Stability of $E_{1}\left(Q / v_{1}, 0, q / \nu_{3}, 0\right)$}

Consider the following jacobian matrix $J_{1}$ corresponding to $E_{1}$

$$
J_{1}=\left[\begin{array}{cccc}
-v_{1} & -\beta_{1} \frac{Q}{v_{1}} & 0 & 0 \\
0 & \left.\frac{\beta_{2} Q v_{3}}{v_{1}\left(\alpha_{1} v_{3}+C_{0} v_{3}-q\right)}\right)^{-v_{2}} & 0 & 0 \\
0 & 0 & -v_{3} & 0 \\
0 & 0 & 0 & -v_{4}
\end{array}\right]
$$


Eigen values are $\lambda_{1}=-v_{1}, \lambda_{2}=-v_{3}, \lambda_{3}=-v_{4}$, and $\lambda_{4}=\frac{\beta_{2} Q v_{3}-v_{1} v_{2}\left(\alpha_{1} v_{3}+C_{0} v_{3}-q_{0}\right)}{v_{1}\left(\alpha_{1} v_{3}+C_{0} v_{3}-q_{0}\right)}$.

Upon implementing Routh-Hurwitz criteria, all the eigen values of matrix $J_{1}$ must be negative. It is observed that $\lambda_{1}, \lambda_{2}$, and $\lambda_{3}$ are negative. Therefore, $E_{1}$ will be stable only if $\lambda_{4}$ is also negative and thus the following condition is obtained $\left(v_{1} v_{2} v_{3} \alpha_{1}+C_{0} v_{1} v_{2} v_{3}\right)>\left(\beta_{2} Q v_{3}+v_{1} v_{2} q_{0}\right)$.

\subsection{Case 2: Stability of $E_{2}\left(N^{\prime}, A^{\prime}, C^{\prime}, 0\right)$}

Corresponding to the point $E_{2}$, jacobian matrix $J_{2}$ is obtained.

$$
J_{2}=\left[\begin{array}{cccc}
-a_{11} & -\beta_{1} N^{\prime} & 0 & 0 \\
a_{21} & a_{22} & a_{23} & -\alpha_{2} A^{\prime} \\
0 & 0 & -v_{3} & 0 \\
0 & 0 & 0 & a_{44}
\end{array}\right]
$$

where $a_{11}=\alpha_{1}+\beta_{1} A^{\prime}$. and $a_{22}=\frac{\beta_{2} N^{\prime}}{\alpha_{1}+C_{0}-C^{\prime}}-v_{2}$.

Upon substituting the values of $N^{\prime}$ and $C^{\prime}$ we get

$$
a_{22}=0, a_{21}=\frac{\beta_{2} A^{\prime}}{\alpha_{1}+C_{0}-C^{\prime}}, a_{23}=\frac{\beta_{2} N^{\prime} A^{\prime}}{\left(\alpha_{1}+C_{0}-C^{\prime}\right)^{2}} \text {, and } a_{44}=\frac{\alpha_{3} A^{\prime}}{\alpha_{4}+C_{0}-C^{\prime}}-v_{4} \text {. }
$$

Characteristic equation corresponding to the above jacobian $J_{2}$ is

$$
\left(-v_{3}-\lambda\right)\left(\frac{\alpha_{3} A^{\prime}}{\alpha_{4}+C_{0}-C^{\prime}}-v_{4}-\lambda\right)\left(\lambda^{2}+a_{11} \lambda+a_{21} N^{\prime} \beta_{1}\right)=0
$$

The eigen values of "(5)" are given by the following equations

$$
\lambda_{1}=-v_{3}, \quad \lambda_{2}=\frac{\alpha_{3} A^{\prime}}{\alpha_{4}+C_{0}-C^{\prime}}-v_{4}=-\left[\frac{v_{4}\left(\alpha_{4}+C_{0}-C\right)-\alpha_{3} A^{\prime}}{\left(\alpha_{4}+C_{0}-C\right)}\right] .
$$

Consider $\left(\lambda^{2}+a_{11} \lambda+a_{21} N^{\prime} \beta_{1}\right)=0$, which gives the following values

$$
\begin{gathered}
\lambda_{3}=\frac{-a_{11}+\sqrt{a_{11}{ }^{2}-4 a_{21} N^{\prime} \beta_{1}}}{2}=\frac{-\left(a_{11}-\sqrt{a_{11}{ }^{2}-4 a_{21} N^{\prime} \beta_{1}}\right)}{2}, \text { and } \\
\lambda_{4}=\frac{-a_{11}-\sqrt{{a_{11}{ }^{2}-4 a_{21} N^{\prime} \beta_{1}}^{2}} .}{2}
\end{gathered}
$$

Upon applying Routh-Hurwitz criteria, it is found that all the eigenvalues with respect to $E_{2}$ are negative. Therefore, the equilibrium point $E_{2}$ is locally asymptotically stable.

4.3. Case 3: Stability of $E_{3}\left(N^{*}, A^{*}, C^{*}, F^{*}\right)$ 
Consider the Jacobian matrix $J_{3}$ corresponding to the point $E_{3}$

$$
J_{3}=\left[\begin{array}{cccc}
-a_{11} & -\beta_{1} N^{*} & 0 & 0 \\
a_{21} & 0 & a_{23} & -\alpha_{2} A^{*} \\
0 & 0 & -v_{3} & 0 \\
0 & a_{42} & a_{43} & a_{44}
\end{array}\right]
$$

The characteristic equation is $\left(-v_{3}-\lambda\right)\left[-\lambda^{3}-a_{11} \lambda^{2}-\left(\alpha_{2} v_{4} F^{*}+a_{21} N^{*} \beta_{1}\right) \lambda-a_{11} \alpha_{2} v_{4} F^{*}\right]=0$.

Therefore, one value of $\lambda$ is $\lambda_{1}=-v_{3}$ which is negative. Now consider the following cubic equation

$$
\lambda^{3}+k_{1} \lambda^{2}+k_{2} \lambda+k_{3}=0
$$

where $k_{1}=a_{11}>0, k_{2}=\alpha_{2} v_{4} F^{*}+a_{21} N^{*} \beta_{1}>0, k_{3}=a_{11} \alpha_{2} v_{4} F^{*}>0$, and $k_{1} k_{2}-k_{3}=a_{11} a_{21} N^{*} \beta_{1}>0$.

Upon implementing Routh-Hurwitz criteria it is shown that the coefficients of $\lambda^{2}, \lambda$, and the constant term in the above cubic equation are positive and the product of coefficients of $\lambda^{2}$ and $\lambda$ is greater than the constant term. This shows that all the eigen values of jacobian $J_{3}$ are negative and thus the equilibrium point $E_{3}$ is asymptotically stable. The dynamic behavior of point $E_{3}$ is analyzed by using Liapunov's direct method in the following theorem.

\section{Nonlinear Stability Analysis of $E_{3}$}

Theorem: The equilibrium point $E_{3}$ is nonlinearly stable if the following conditions are satisfied:

$$
\begin{gathered}
{\left[\frac{n_{1} \beta_{2} v_{3}}{\left(\alpha_{1} v_{3}+C_{0} v_{3}-q\right)}-\beta_{1} N^{*}\right]^{2}<\frac{2 n_{1} v_{1} \alpha_{2}}{3}\left[\frac{n_{1} \beta_{2} N^{*}}{\left(\alpha_{1} v_{3}+C_{0} v_{3}-q\right)\left(\alpha_{1}+C_{0}-C^{*}\right)}\right]^{2} v_{3}<\frac{n_{1} n_{2} \alpha_{2}}{3},} \\
{\left[\frac{n_{3} \alpha_{2} v_{3}}{\left(\alpha_{4} v_{3}+C_{0} v_{3}-q\right)}\right]^{2}<\frac{n_{1}^{2} \alpha_{2}^{2}}{6} \text {, and }\left[\frac{n_{3} \alpha_{3} A^{*}}{\left(\alpha_{4} v_{3}+C_{0} v_{3}-q\right)\left(\alpha_{4}+C_{0}-C^{*}\right)} v_{3}<\frac{n_{1} n_{2} \alpha_{2}}{2} .\right.}
\end{gathered}
$$

Proof: Let us consider the positive definite function

$$
V=\frac{1}{2}\left(N-N^{*}\right)^{2}+n_{1}\left(A-A^{*}-A^{*} \ln \frac{A}{A^{*}}\right)+\frac{1}{2} n_{2}\left(C-C^{*}\right)^{2}+n_{3}\left(F-F^{*}-F^{*} \ln \frac{F}{F^{*}}\right)
$$

where $n_{1}, n_{2}$ and $n_{3}$ are positive constants, to be chosen appropriately. Let us consider the derivative

$$
\begin{gathered}
\frac{d V}{d t}=\left(N-N^{*}\right) \frac{d N}{d t}+n_{1} \frac{\left(A-A^{*}\right)}{A} \frac{d A}{d t}+n_{2}\left(C-C^{*}\right) \frac{d C}{d t}+n_{3} \frac{\left(F-F^{*}\right)}{F} \frac{d F}{d t} \\
\frac{d V}{d t}=Y_{1} \frac{d N}{d t}+n_{1} \frac{Y_{2}}{A} \frac{d A}{d t}+n_{2} Y_{3} \frac{d C}{d t}+n_{3} \frac{Y_{4}}{F} \frac{d F}{d t}
\end{gathered}
$$


where $Y_{1}=\left(N-N^{*}\right), Y_{2}=\left(A-A^{*}\right), Y_{3}=\left(C-C^{*}\right), Y_{4}=\left(F-F^{*}\right)$

Upon substituting the values of $d N / d t, d A / d t, d C / d t$ and $d F / d t$ from the model "(1)-(4)" and then applying some algebraic manipulations by using the inequality $x^{2}+y^{2} \geq 2 x y, d V / d t$ is reduced into following inequality

$$
\begin{aligned}
& \frac{d V}{d t} \leq-\beta_{1} A Y_{1}^{2}-\frac{1}{2} 2 v_{1} Y_{1}^{2}+Y_{1} Y_{2}\left[\frac{n_{1} \beta_{2}}{\left(\alpha_{1}+C_{0}-C\right)}-\beta_{1} N^{*}\right]-\frac{1}{2} \frac{n_{1} \alpha_{2}}{3} Y_{2}^{2}-\frac{1}{2} \frac{n_{1} \alpha_{2}}{3} Y_{2}^{2} \\
& +\frac{n_{1} \beta_{2} N^{*}}{\left(\alpha_{1}+C_{0}-C\right)\left(\alpha_{1}+C_{0}-C^{*}\right)} Y_{2} Y_{3}-\frac{1}{2} n_{2} v_{3} Y_{3}^{2}-\frac{1}{2} \frac{n_{1} \alpha_{2}}{3} Y_{2}^{2}+\frac{n_{3} \alpha_{3}}{\left(\alpha_{4}+C_{0}-C\right)} Y_{2} Y_{4}-\frac{1}{2} \frac{n_{1} \alpha_{2}}{2} Y_{4}^{2}-\frac{1}{2} n_{2} v_{3} Y_{3}^{2} \\
& +\frac{n_{3} \alpha_{3} A^{*}}{\left(\alpha_{4}+C_{0}-C\right)\left(\alpha_{4}+C_{0}-C^{*}\right)} Y_{3} Y_{4}-\frac{1}{2} \frac{n_{1} \alpha_{2}}{2} Y_{4}^{2} . \\
& \quad \frac{d V}{d t} \leq-\beta_{1} A Y_{1}^{2}-\frac{1}{2} P_{11} Y_{1}^{2}+P_{12} Y_{1} Y_{2}-\frac{1}{2} P_{22} Y_{2}^{2}-\frac{1}{2} P_{22} Y_{2}^{2}+P_{23} Y_{2} Y_{3}-\frac{1}{2} P_{33} Y_{3}^{2}-\frac{1}{2} P_{22} Y_{2}^{2}+P_{24} Y_{2} Y_{4} \\
& \quad-\frac{1}{2} P_{44} Y_{4}^{2}-\frac{1}{2} P_{33} Y_{3}^{2}+P_{34} Y_{3} Y_{4}-\frac{1}{2} P_{44} Y_{4}^{2} .
\end{aligned}
$$

where

$$
\begin{gathered}
P_{11}=2 v_{1}, P_{22}=\frac{n_{1} \alpha_{2}}{3}, P_{33}=n_{2} v_{3}, P_{44}=\frac{n_{1} \alpha_{2}}{2}, P_{23}=\frac{N^{*} n_{1} \beta_{2}}{\left(\alpha_{1}+C_{0}-C\right)\left(\alpha_{1}+C_{0}-C^{*}\right)}, \\
P_{24}=\frac{n_{3} \alpha_{3}}{\left(\alpha_{4}+C_{0}-C\right)}, P_{34}=\frac{n_{3} \alpha_{3} A^{*}}{\left(\alpha_{4}+C_{0}-C\right)\left(\alpha_{4}+C_{0}-C^{*}\right)}, \text { and } P_{12}=\frac{n_{1} \beta_{2}}{\left(\alpha_{1}+C_{0}-C\right)}-\beta_{1} N^{*} .
\end{gathered}
$$

Hence, the sufficient conditions for $d V / d t$ to be negative definite are given by the following inequalities

$$
P_{12}^{2}<P_{11} \cdot P_{22}, P_{23}^{2}<P_{33} \cdot P_{22}, P_{24}^{2}<P_{44} \cdot P_{22}, \text { and } P_{34}^{2}<P_{33} \cdot P_{44} \cdot
$$

Hence, $V$ is a Liapunov's function with respect to $E_{3}$.

\section{Numerical Simulation}

The validity of the proposed model for verifying the results obtained by stability analysis is carried out. The following values for various parameters of the model are taken from the literature [16].

$$
\begin{gathered}
Q=3, \beta_{1}=0.5, \beta_{2}=0.35, v_{1}=0.1, v_{2}=0.009, v_{3}=3, v_{4}=0.01 \\
\alpha_{1}=0.51, \alpha_{2}=0.41, \alpha_{3}=0.33, \alpha_{4}=0.3, q=24, C_{0}=30
\end{gathered}
$$

The values obtained for $E_{3}\left(N^{*}, A^{*}, C^{*}, F^{*}\right)$ are $N^{*}=6.8191, A^{*}=0.6799, C^{*}=7.9994$, and $F^{*}=0.2367$. These points are further checked for the conditions of existence of equilibrium point $E_{3}$. This gives

$$
\alpha_{4} v_{3}+C_{0} v_{3}-q=66.9>0,\left(\alpha_{4}+C_{0}-C^{*}\right)=22.3>0, \beta_{2} N^{*}-v_{2}\left(\alpha_{1}+C_{0}-C^{*}\right)=2.19491>0, \text { and }
$$




$$
\left(\alpha_{1}+C_{0}-C^{*}\right)=22.51>0
$$

It shows that the non negativity conditions are satisfied and thus $E_{3}$ is nonlinear stable.

\section{Conclusion}

In this paper, a mathematical model is proposed and analyzed to understand the effect of the growth of algae population in aquatic body in the presence of nutrients and its impact on the survival of fish population. Three equilibrium points are identified and the criteria of their existence are obtained. By applying stability analysis, it is shown that all the feasible equilibrium points are locally asymptotically stable if they satisfy these conditions. The nonlinear stability behavior of the point $E_{3}$ is studied by applying Liapunov's direct method. By numerical solution of the model, it is shown that $E_{3}$ is a nonlinear stable for the considered values of parameters. This shows that the proposed model is valid and can effectively estimate the effect of depleting dissolved oxygen on the survival of fish population in aquatic body in the presence of nutrients.

\section{References}

[1] Streeter, M. W., \& Phelps, E. E. (1925). A Study of Pollution and Natural Purification of the Ohio River (pp. 146-150). U.S. Public Health Service Bill.

[2] Dobbins, W. E. (1964). BOD and oxygen relationship in streams. J. San. Eng. Div., 53-78.

[3] O'Conner, D. J. (1967). The temporal and spatial distribution of dissolved oxygen in steams. Water Resour.Res, 3, 65-79.

[4] Misra, A. K. (2008). Mathematical modeling and analysis of the depletion of dissolved oxygen in water bodies. Nat. Acad. Sci. India. A, 78(iv), 331-340.

[5] Freedman, H. I., \& So, J. W. H. (1985). Global Stability and persistence of simple food chains. Math, Biosciences, 76, 69-86.

[6] Gates, W. E., Marler, J. T., \& Westfield. J. D. (1969). The application of bacterial process kinetics in stream simulation and stream analysis. Water Research, 3, 663-686.

[7] Hale, J. K. (1969). Ordinary Differential Equations. Wiley Interscience, Newyark.

[8] Jorgensen, S. E. (1988). Fundamental of Ecological Modeling. Elsevier Science Publisher.

[9] Arnold, E. M., \& Voss, D. A. (1981). Numerical behavior of zooplankton, phytoplankton and phosphorus system. Ecol. Modeling, 13, 183-193.

[10] Gates, W. E., Marler, J. T., \& Westfield, J. D. (1969). The application of bacterial process kinetics in stream simulation and stream analysis. Water Research, 3, 663-686.

[11] Shukla, J. B., et al. (2001). Existence and survival of two competing species in a polluted environment. J. Biol. Syst., 9, 89-103.

[12] Shukla, J. B., et al. (1996). Effect of changing habitant on survival of species. Ecol. Model., 87, 205-216.

[13] Dhar, J., et al. (2010). Role of toxin producing phytoplankton on a plankton ecosystem. Nonlinear Analysis: Hybrid Systems, 4, 496-502.

[14] Swati, K., et al. (2009). Effect of soil pollutant on the plant-herbivore interacting system incorporating nutrient cycling: A mathematical model. J. Scientific Research, 53, 163-174.

[15] Edwards, A. M., et al. (1999). Zooplankton mortality and dynamical behavior of plankton population models. Bul. Math. Biol., 61, 303-339.

[16] Swati, K., et al. (2013). Modelling effect of the depleting oxygen on the existence of interacting planktonic population. Elixir Appl. Math., 55, 12739-12742. 


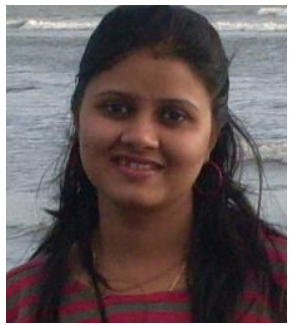

Shikha Chaturvedi was born at Etawah (U.P.), India on July 12, 1981. She got the bachelor of science (B.Sc.) in mathematics, physics, chemistry from C.S.J.M. University, UP, India in 2000, and got the master of science (M.Sc.) in applied mathematics from Indian Institute of Technology, Kanpur, India in 2003. Now she is pursuing her Ph.D. degree in applied mathematics from Shri J.J.T., University, Rajasthan, India.

She has 12 years of teaching experience in various Engineering Institutes. Currently she is working as an assistant professor in Fr. C. Rodrigues Institute of Technology, Navi Mumbai, Maharashtra, India. She has worked as a research associate in Indian Institute of Technology, Kanpur, India. She published some articles.

Her area of research is mathematical modeling. Ms. Chaturvedi has got some professional awards and achievements.

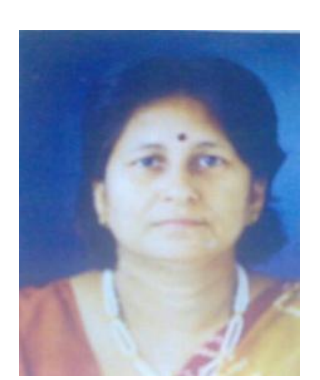

Prabha S. Rastogi was born at Lucknow (U.P.), India on October 21, 1954. She got the B.Sc., M.Sc., and PhD degrees in mathematics from Lucknow, UP, India in 1973, 1975, 1985 respectively.

She has 31 years of teaching experience as Professor in Bhavans College, Andheri, Mumbai, India. Currently she is associated with Shri JJT University, Rajasthan, India.

Dr Prabha has given her valuable guidance to many research scholars. She is on editorial board of research journal Research Dimensions and core committee member of registered International Research society Prerna Society of Technical Education and Research, New Delhi. She is also associated with Reliable Publications (educational publishers). 